\title{
Mannewitz, Tom (2020): Das erste Forschungsprojekt. Karte und Kompass für junge Politik- und SozialwissenschaftlerInnen
}

\author{
Baden-Baden: Nomos. 344 Seiten. $29 €$
}

\author{
Julia Reuschenbach $(1)$
}

Angenommen: 29. Juni 2021 / Online publiziert: 19. Juli 2021

(C) Der/die Autor(en) 2021

Lehrbücher sind auf dem Reputationsmarkt der Politikwissenschaft keine besonders hoch gehandelte Ware. Trotzdem arbeiten AutorInnen wie Tom Mannewitz über viele Monate an diesen Werken, die in erster Linie Studierenden zugutekommen, selten aktuelle Fachdiskussionen bereichern und meist kaum bis gar nicht von FachkollegInnen wahrgenommen werden. Für ein solches Projekt muss man daher sein, was wohl häufig ein/e ÜberzeugungstäterIn genannt wird. Mannewitz ist ein solcher Überzeugungstäter. Mit seinem Lehrbuch wertschätzt er hochschuldidaktische Fragen der Vermittlung von Methoden- und Forschungskompetenzen und damit den Stellenwert politikwissenschaftlicher Hochschullehre insgesamt. Der Autor lässt über 344 Seiten hinweg deutlich werden, wie sehr er am Forschungs- und Lernerfolg seiner Studierenden interessiert ist. Ein potenzieller Erfolg, der am Ende auch immer BetreuerInnen selbst zugutekommen kann, die studentische Forschungsprojekte begleiten und bewerten müssen. Mannewitz unternimmt mit diesem Lehrbuch überzeugend den Versuch, Studierende mit den Methoden der empirischen Sozialforschung aus dezidiert politikwissenschaftlicher Perspektive anwendungsorientiert und entlang studentischer Forschungsprojekte vertraut zu machen. Der Band ist damit mehr als ein klassisches Lehrbuch, sondern auch Begleiter und Ratgeber und praktischer Leitfaden im Forschungsprozess.

Die insgesamt acht Kapitel umfassende Publikation führt Studierende in den ersten drei Kapiteln an die zentralen Botschaften des Bandes heran: (1) Noch ein Methodenbuch ..., (2) Wissenschaftstheorie in 60 Minuten und (3) Ohne geht's nicht: das kleine Einmaleins empirischer Sozialforschung. Beginnend mit Kapitel 4 geht es anschließend um die Forschungsplanung, die Datenerhebung (Kapitel 5) und die

\footnotetext{
Julia Reuschenbach $(\bowtie)$

Institut für Politische Wissenschaft und Soziologie, Rheinische Friedrich-Wilhelms-Universität,

Bonn, Deutschland

E-Mail: julia.reuschenbach@uni-bonn.de
} 
Datenauswertung (Kapitel 6). Das Lehrbuch schließt mit Hinweisen zur Präsentation der eigenen Forschungsergebnisse (Kapitel 7) und zehn ,goldenen Regeln“ zum Schreiben einer (ersten) wissenschaftlichen Arbeit (Kapitel 8).

Sprachlich verständlich und in einer sehr anschaulichen Darstellung lässt der Autor vor allem im Kapitel 2 keinen Zweifel daran, dass für alle Studierenden der Politikwissenschaft Methodenkompetenzen, aber auch wissenschaftstheoretisches Grundlagenwissen schlichtweg unabdingbar sind. Mannewitz liefert dabei eine absolut überzeugende Einordnung auf eine Kernfrage des Studiums, die etwas salopp lauten könnte: „Was mache ich hier eigentlich?“ - eine Frage, die sich Studierende im Laufe des Studiums vermutlich nicht nur einmal stellen und die gerade bei ersten Schritten auf dem Weg zu Qualifikationsarbeiten immer wieder auftaucht. Besonders hervorzuheben sind dabei die durch den Autor vorgenommene Vergegenwärtigung der „Physik des Politischen“ (S. 19), die Betonung von Kritikfähigkeit als ,wichtigste Zutat für wissenschaftlichen Fortschritt“" (S. 22) und die wohl nicht von allen in der Disziplin geteilte Ansicht, dass es sich keineswegs um fehlende Wissenschaftlichkeit, sondern vielmehr um ein Kompliment für den Autor handelt, wenn Fachfremde einen wissenschaftlichen Text begreifen (S. 21). Wo dieser konstruktive Appell Studierende womöglich von blumiger und komplizierter Sprache abhalten kann, sind andere Passagen des Bandes geeignet, Verunsicherung bei Studierenden zu erzeugen. So bleibt offen, ob Studierende nach der Lektüre der ausführlichen Textpassagen zur Werturteilsbildung (vor allem S. 40-41) noch genügend Zutrauen haben, sich auf dieses verminte Terrain zu wagen. Dies gilt erst recht vor dem Hintergrund des Hinweises, wer in Seminaren regelmäßig ,,volle Breitseite bekomme, sollte sich genau fragen, ob er Werturteile in schriftlichen Analysen entfalten will“ (S. 60). So sehr die grundlegende Botschaft des Autors nachvollziehbar erscheint (Werturteilsbildung ist ein anspruchsvolles, bisweilen umstrittenes Vorgehen und sollte nicht am Anfang studentischer Forschungen stehen), so sehr ist die Fähigkeit zur Werturteilsbildung doch abhängig von intensiver Übung, gegenseitiger Kritik und gemeinsamer Diskussion. Und wo sollte diese besser und in besonderem Umfang stattfinden als in der Hochschullehre und im Austausch mit Dozierenden? Ganz anders klingt Mannewitz hingegen im Abschnitt $4.3 \mathrm{zu}$ Themen, Fragen, Hypothesen und im Abschnitt $4.4 \mathrm{zu}$ Forschungsstand, theoretischer Ansatz und Variablenauswahl, in denen er Studierenden in der schwierigen Phase vor Beginn eines Forschungsprojekts hilfreiche Einordnungen und Tipps anbietet und anhand konkreter Beispiele die Unterschiede in den Anforderungen verschiedener Qualifikationsphasen verdeutlicht (S. 107-115).

Mit den anschließenden Kapiteln 5 und 6 zeigt der Band seine Stärken in Form von Praxisnähe und Anschaulichkeit. Hand in Hand können die LeserInnen ihren Forschungsprozess gemeinsam mit Mannewitz durchschreiten und sich passgenau heraussuchen, was für ihre Arbeit besonders relevant erscheint. So bietet der Band Einblick in klassische Erhebungsmethoden, von Befragungen über Beobachtungen, Inhaltsanalysen und Experimente bis hin zu nichtreaktiven Verfahren. Dabei hinterfragt Mannewitz gemeinsam mit seinen LeserInnen die Auswahl der Erhebungsmethoden und hilft über mögliche Schwierigkeiten hinweg, indem er mögliche Probleme und Fehlschlüsse darstellt. Manche Darstellungen fallen dabei ausführlicher aus, etwa die zu standardisierten Interviews (S. 147-158) oder zu Experimenten 
(S. 192-198), andere wie jene zu Experteninterviews (S. 138-140) deutlich kürzer. Klar ist dabei aber auch, dass sich, wer Experteninterviews führen möchte, nicht allein auf die knappen Ausführungen bei Mannewitz berufen kann. Dies macht der Band von Beginn an deutlich und transparent. Er baut zugleich aber Brücken in mögliche Vertiefungsebenen, etwa durch kurze Zusammenfassungen und kommentierte wie wohldosierte Literaturtipps am Ende jedes Kapitels. So ist in diesem Lehrbuch ein Blick in aller Kürze ebenso möglich wie gezielte Wiederholungseinheiten. Insgesamt ist dieses Buch damit nicht nur etwas für Studierende, die an einem ersten Forschungsprojekt arbeiten, sondern auch etwas für all jene, die Grundlagen erneut in Erinnerung rufen möchten oder im Rahmen ihres Studiums mit den Methoden der empirischen Sozialforschung nur wenig in Berührung gekommen sind. Abschließend ist darauf hinzuweisen, dass der Band vielfältige Tipps zur Nutzung von Suchmaschinen, Onlinedatenbanken, Literaturverwaltungsprogrammen und Analysesoftware beinhaltet. Auch wenn solche Hinweise den technischen Fortschritt meist nicht allzu lange überdauern, tut dies der Qualität dieses Lehrbuchs keinen Abbruch.

Funding Open Access funding enabled and organized by Projekt DEAL.

Open Access Dieser Artikel wird unter der Creative Commons Namensnennung 4.0 International Lizenz veröffentlicht, welche die Nutzung, Vervielfältigung, Bearbeitung, Verbreitung und Wiedergabe in jeglichem Medium und Format erlaubt, sofern Sie den/die ursprünglichen Autor(en) und die Quelle ordnungsgemäß nennen, einen Link zur Creative Commons Lizenz beifügen und angeben, ob Änderungen vorgenommen wurden.

Die in diesem Artikel enthaltenen Bilder und sonstiges Drittmaterial unterliegen ebenfalls der genannten Creative Commons Lizenz, sofern sich aus der Abbildungslegende nichts anderes ergibt. Sofern das betreffende Material nicht unter der genannten Creative Commons Lizenz steht und die betreffende Handlung nicht nach gesetzlichen Vorschriften erlaubt ist, ist für die oben aufgeführten Weiterverwendungen des Materials die Einwilligung des jeweiligen Rechteinhabers einzuholen.

Weitere Details zur Lizenz entnehmen Sie bitte der Lizenzinformation auf http://creativecommons.org/ licenses/by/4.0/deed.de. 